\title{
Multi-beam Bathymetry Method Based on Low Autocorrelated Sidelobe Binary Sequences and Its Implementation on FPGA
}

\author{
Li Haisen ${ }^{1,2, ~ a, ~ L u ~ D o n g ~}{ }^{1,2, ~ b}$, Chen Baowei $^{1,2, c^{\star}}$ and Zhou Tian ${ }^{1,2, d}$ \\ ${ }^{1}$ Acoustic Science and Technology Laboratory, Harbin Engineering University, Harbin 150001, \\ China; \\ ${ }^{2}$ College of Underwater Acoustic Engineering, Harbin Engineering University, Harbin 150001, China \\ a hsenli@126.com, ${ }^{b}$ ludong0215@126.com, ${ }^{c}$ cbwwin@163.com, ${ }^{\mathrm{d}}$ zhoutian@hrbeu.edu.cn
}

Keywords: multi-beam; pulse compression; FPGA; low autocorrelated sidelobe binary sequences

\begin{abstract}
Given the problem that the range resolution is reduced as a result of overcoming the reduction of SNR (Signal to Noise Ratio) of echo signal only by enlarging the pulse length due to deeper water depth and worsened turbidity in multi-beam bathymetry of single frequency pulse, multi-beam bathymetry method based on low autocorrelated sidelobe binary sequences was proposed. Firstly, the principles of multi-beam bathymetry based on low autocorrelated sidelobe binary sequences were discussed, then the SNRs and resolution performances of narrow pulse method and low autocorrelated sidelobe binary sequences method were compared by means of software simulation, and it was verified by a shallow water multi-beam bathymetric system with wide coverage in a test on Songhua Lake. The results show that this method effective increases SNR and improves bathymetry quality at the premise of ensuring the bathymetry accuracy. Finally, a rapid calculation structure based on FPGA was proposed. The results show that this structure which has comparatively small calculation amount makes it possible to realize real-time processing.
\end{abstract}

\section{Introduction}

In conventional shallow water multi-beam bathymetric systems, the probe signal is often single frequency narrow pulse. However, with deeper measuring depth and worsened turbidity, the SNR of echo signal from the seabed will decrease drastically. As a result, the submarine topography cannot be calculated accurately. In order to increase SNR, the pulse length of the signal sent will have to be increased, but this is realized at the cost of bathymetry accuracy. Bathymetry accuracy and SNR becomes a pair of contradictions. The probe signal for pulse compression is often linear FM signal and coded signal. The research on the former is thorough. However, the convolution involves in numerous multiply-accumulate calculations in practical application, which may result in large calculation amount, in particularly in multi-beam bathymetric system, where the calculation amount is unacceptable as there are the data of dozens of channels to be processed simultaneously. The convolution of two-phase coded signal is only multiply-accumulate calculation of single-bit signal, and the multiplication can be omitted by processing, leaving additive operation only. So the calculation amount is easily acceptable. In recent years, research on coded signal has witnessed sufficient development. Many coded signals are featured by the characteristics of pulse compression, however, in multi-beam bathymetry, enlarged sidelobe of pulse compression will cause the bathymetric algorithm based on the phase to be seriously interfered by the decoherence of the base line [1]. Therefore, when choosing probe signal, coded signal with lower autocorrelated sidelobe should be chosen as far as possible. M. Amin Nasrabadi researched the method for fast searching the lowest autocorrelated sidelobes [2, 3], Nunn C J summarized the then known optimal low autocorrelated sidelobe binary sequences with encoding length ranging from 71 to 105 [4]. Kai-Uwe Schmidt proposed the boundary of minimum peak sidelobe theory [5], and Anatolii N. Leukhin further researched the method for exhaustive searching minimum peak sidelobes, he found the searching minimum peak sidelobes in the range of 74 lengths [6]. Based on the predecessors' basis, 
shallow water multi-beam bathymetry method based on low autocorrelated sidelobe binary sequences was proposed in this paper.

In this paper, low autocorrelated sidelobe binary sequence was presented first, then it was introduced into shallow water multi-beam bathymetry method, and the effectiveness of this method was verified by means of software simulation and the data from lake test. The results show that this method effectively increases SNR and improves bathymetry quality at the premise of ensuring bathymetry accuracy. Finally, a rapid calculation structure based on FPGA was proposed, which made it possible to realize real-time processing of this method.

\section{Principles of the algorithm}

With the proposal of low autocorrelated sidelobe binary sequences, M. Amin Nasrabadi proposed an exhaustive searching method [2]. Some achievements have been achieved in recent years. However, with the increase of code length, the calculation amount for searching increases drastically, the code has increased to 2 bits from 105 bits, its PSL is as shown in Fig. 1. When it reaches 105 bits, PSL (peak sidelobe level) will reach $26.4444 \mathrm{~dB}$.

Narrow pulse signal will be attenuated due to long-distance transmission and turbid environment as the energy it carries is small. The time bandwidth product of the coded signal with consistent code element width and narrow pulse is the number of code elements, its GSNR will be its time bandwidth product after passing matching filter. This means that the longer the code length is, the more SNR will be improved. Meanwhile, after compression, its main lobe width is still the code element width, so it has the same resolution as the narrow pulse signal, i.e., the SNR is improved at the premise of keeping the resolution unchanged.

However, introducing coded signal will bring sidelobes in distance, which will cause the bathymetric algorithm based on the phase to be seriously interfered by the decoherence of the base line [1]. The longer the duration of sidelobe is, the larger the influence of sidelobe on phase method will be, so it needs to lower the sidelobe and reduce the pulse length as far as possible. This is contradictive to improving SNR. In practical application, it needs to determine it through analysis based on specific conditions. When the SNR of the echo signal is too low to be detected, the detection effect can be improved by increasing the number of code elements, although this may lose some phase measurement accuracy.

Apart from sidelobes, after the coded signal passes the matching filter, its main lobe part can approximately equal to CW pulse signal. Low autocorrelated sidelobe codes which have lower sidelobes can be considered to be ignored when designing topography calculating algorithm. Based on this, the proposed bathymetry method includes the following steps:

1) Signals of low autocorrelated sidelobe binary sequences which pass binary phase shift keying are sent by the transducer to form a strip vertical to the course on the seabed;

2) The echo signals scattered from the seabed target are converted into multi-channel complex signals through reception linear array, signal conditioning, undersampling and IQ demodulation;

3) Coded pulse compression is carried out for the real part and the imaginary part of the complex signals to obtain the complex signals after pulse compression;

4) The sidelobes of the signals with compressed pulse are comparatively low, so the signals can be deemed as CW pulse signal when ignored, and they can be directly sent to the beam former;

5) Topography detection is carried out by means of WMT(weight mean time)or phase methods according to the beam form results.

\section{Simulation}

Because of concerning about the level of sidelobes, the length of sidelobe and SNR, low autocorrelation sequence which length is 82 (Hex: 3CB25D380CE3B7765695F) is selected as the detection signal. The sequence is applied to the bathymetric multi-beam echo sounders, $19.08 \mathrm{~dB}$ SNR can be improved theoretically, while keeping a high resolution. To convenient the simulation 
study, flat seafloor model is selected. Simulations have been conducted in the FIELD II[7], where the emission array element number is 56 and spacing is $5.95 \mathrm{~mm}$, the receiving array element number is 80 and spacing is $4.166 \mathrm{~mm}$, center frequency is $180 \mathrm{kHz}$, sampling rate is $140 \mathrm{kHz}$, and measured target is $30 \mathrm{~m}$ flat seafloor. There are two kinds of signals are used as the transmitted signal $(0.1 \mathrm{~ms}$ $\mathrm{CW}$ pulse signal and low autocorrelation sequence signal modulated by the $0.1 \mathrm{~ms}$ symbol, and the sequence length is 82). There are two kinds of echo signal is superimposed on the same power Gaussian white noise, and the processed results are shown in Fig. 1. The simulation results of CW pulse are shown in Fig. 1(a), the simulation results of proposed are shown in Fig. 1(b) (detection results are represented by the black point). Fig. 1(a) clearly shows that terrain information cannot be represented at the edge of the beam due to lower SNR. However, Fig. 1(b) clearly shows that terrain information is expressed very clearly even at the edge of the beam, because the SNR is improved by proposed method. Through statistical analysis, it can be seen that SNR is improved by 18.91dB while keeping a high resolution.
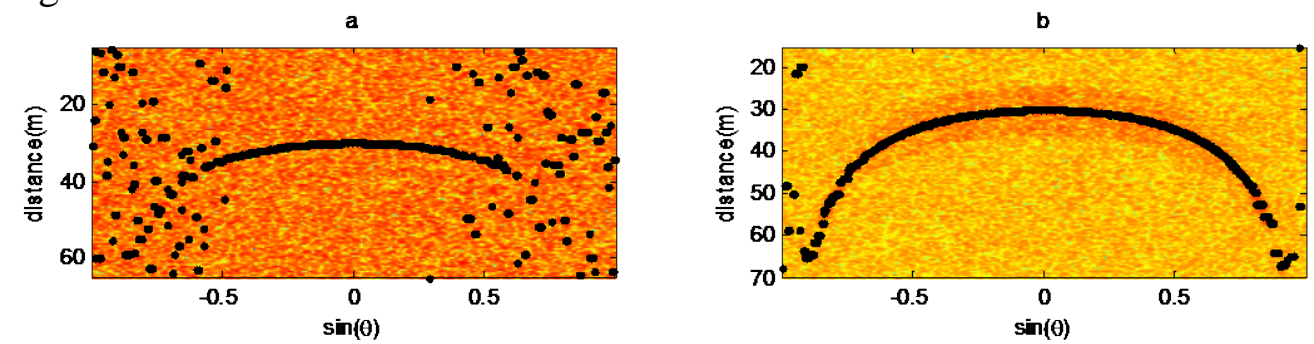

Fig. 1. Simulation results of CW pulse (a) and low autocorrelation sequence (b)

\section{Field data and experimental results}

To fully verify the effectiveness of the method, the domestic shallow-water wide-coverage bathymetric multi-beam echo sounders is selected as the experimental device to collect datas in Jilin Songhua Lake (50m water depth), its transmitting and receiving array has the same parameters with simulation. There are two signals with the same instantaneous power are used as the transmitted signal. The processed results of two echo signals are shown in Fig. 2. The results of CW pulse are shown in Fig. 2(a), the results of proposed are shown in Fig. 2(b) (detection results are represented by the black point). It can be seen that this method has a higher SNR (improved 15.82dB), while keeping a high resolution and improves the quality of sounding effectively.

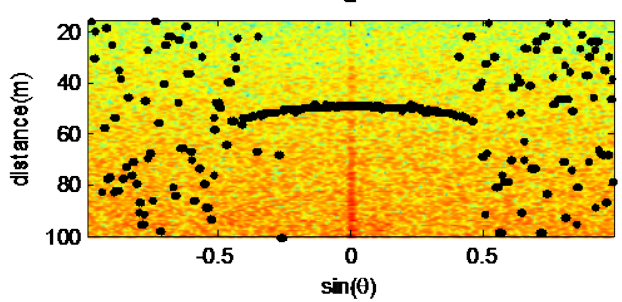

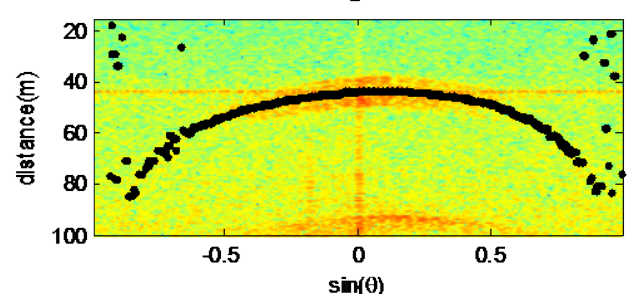

Fig. 2. Test results on the lake of CW pulse (a) and low autocorrelation sequence (b)

\section{Implementation on FPGA}

CW narrow pulse is applied to the conventional bathymetric multi-beam echo sounders, which has a relatively small computational complexity. However, the computational complexity increasing dramatically with the signal length increasing after the pulse compression is introduced. The PM-1 times accumulate operations are required by conventional pulse compression method of coded signal, where the code length is $\mathrm{M}$, the number of sampling points of single symbol duration is $\mathrm{P}(\mathrm{P}=\mathrm{T} * \mathrm{Fs}$ / M), the signal length is $\mathrm{T}$, and the sampling rate is Fs. Comparing to the LFM, although eliminating the multiplication, the pulse compression method still maintains a large computational complexity, especially when the $\mathrm{P}$ is larger, the computational complexity double increase. The computational complexity must be further reduced for real-time applications. Thus, the rapid pulse compression structure of the encoded signal is presented in Fig. 3: 


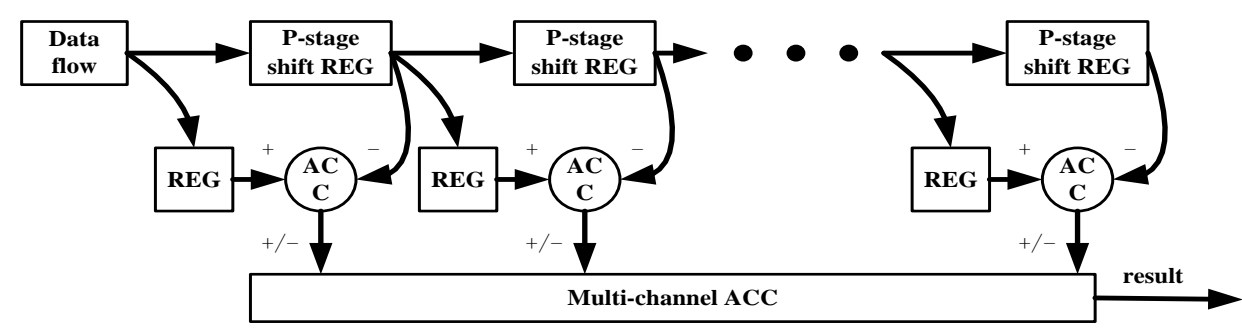

Fig. 3. New pulse compression structure

It can be seen that the method needs only 3M-1 times additions or subtraction at each sampling point in addition to the registers, comparing to the PM-1 times in conventional method, computational complexity is reducing several times, when $\mathrm{P}$ is $14, \mathrm{M}$ is 82 in this paper, computational complexity is only 0.21 times conventional methods. For quick calculation method beamforming has been mentioned in paper[8], this paper will not repeat.

\section{Summary}

In this paper, low autocorrelated sidelobe binary sequences serve as the detection signals for shallow water multi-beam bathymetry based on their characteristics, and the corresponding bathymetric algorithm is proposed. Simulation and analysis on the data from lake test show that this method effective increases SNR and improves bathymetry quality at the premise of ensuring the bathymetry accuracy. Further analysis is carried out on the characteristics of the coded signals, and a simple and rapid calculation structure based on FPGA is proposed, which makes it possible to realize real-time processing of this method and has certain engineering application value.

\section{Acknowledgment}

This work was financially supported by the National Natural Science Foundation of China (Grant No. 41306038, 41327004, 41376103, 41306182 and 61401112), the Specialized Research Fund for the Doctoral Program of Higher Education of China (Grant No. 20112304130003, 20122304120012), the National Laboratory of Underwater Acoustic Technology Foundation of China (Grant No. 9140C200105120C2001), the National Defense Pre-Research Foundation of China (Grant No. 40102), the Fundamental Research Funds for the Central Universities of China (Grant No. HEUCF140501, HEUCF140502).

\section{References}

[1] Vincent P., Maussang F., Lurton X., et al, in: OCEANS - Bergen, 2013 MTS/IEEE, 2013, 1-4.

[2] Nasrabadi M. A., Bastani M. H, in: Radar Systems, 2007 IET International Conference on, Edinburgh, UK, 2007.

[3] Nasrabadi M. A., Bastani M. H, in: Radar, CIE '06. International Conference on, Shanghai, 2006.

[4] Nunn C. J., Coxson G. E, in: IEEE Transactions on Aerospace and Electronic Systems. 2008,44(1): 392-395.

[5] Schmidt K. , in: Information Theory, IEEE Transactions on. 2012, 2512-2515.

[6] Leukhin A. N., Potekhin E. N, in: Microwave Conference (EuMC), 2013 European, Nuremberg, 2013.

[7] Jensen J. A, in: Medical and Biological Engineering and Computing. 1996,34(S1): p351-353.

[8] Haisen L., Dong L., Xiangyu C., et a, in: Instrumentation, Measurement, Computer, Communication and Control (IMCCC), Harbin (2012). 